\title{
SELECTED EXAMPLES OF REFERRING THE EXAMINED STOCHASTIC TECHNICAL STABILITY TO THE ISO STANDARDS
}

\author{
JERZY KISILOWSKI \\ Kazimierz Pulaski University of Technology and Humanities in Radom, Faculty of Transport and Electrical Engineering, \\ Radom, Poland; e-mail: jkisilow@kisilowscy.waw.pl \\ JAROSŁAW ZALEWSKI \\ Warsaw University of Technology, Faculty of of Administration and Social Sciences, Warsaw, Poland \\ e-mail: j.zalewski@ans.pw.edu.pl
}

\begin{abstract}
In this paper, certain examples of comparison between the selected definition of the stochastic technical stability (for motor vehicle models) and the ISO definitions (developed for real vehicles) have been discussed. Being able to refer the examined stability of a mathematical model to the real object may result in verifying the properties of the latter on the basis of model tests, instead of the field trials. Such an attempt has been presented previously, but in a more general approach. The aim of this paper is to present a certain attempt to compare the results obtained for a simulated vehicle model in virtual environment with the specific definitions of stability dedicated to the real motor vehicles. The background of this considerations liesin the examination of the stochastic technical stability, which allows any mechanical system undergo external random disturbances, such as road irregularities.
\end{abstract}

Keywords: motor vehicle stability, ISO norm, comparisons

\section{Introduction}

In the analysis of stability it seems important to choose an appropriate definition which, depending on the ease of interpretation and nature of disturbances, allows analysis of the tested object. The ability to refer the obtained results to real-world objects (e.g. motor vehicle) seems to be important, for which stability criteria are determined according to the ISO standards.

Stability of a nonlinear mathematical model of a motor vehicle, with additional external disturbances, is considered. Disturbances stemming from road irregularities are assumed as a stochastic process, stationary in broader sense as well as globally ergodic, which enabled analysis based on a single trajectory representing motion of the vehicle (Kisilowski and Zalewski, 2008).

For motor vehicles, the stability is adopted in accordance with ISO 8855:1991 which, in comparison to the stochastic technical stability, gives an opportunity to examine the motor vehicle stability on the basis of an exemplary model (described with the use of differential equations) and refer the results to the real one, in real maneuver.

The term "stochastic" has been previously connected to the aspects of stability, e.g. in (Khasminskii, 2012; Kisilowski and Zalewski, 2016), however not related to the ISO standards. Stability was also examined for motor vehicles, e.g. in (Karnopp, 2004), however not combining both the use of the stochastic technical stability and the ability to relate the results to real vehicles.

Certain aspects of vehicle stability were considered previously. For example, in (Chung and Yi, 2006) motor vehicle stability control based on the side slip angle was proposed, as well as the the evaluation of certain control schemes on a virtual test track. In (Doumiati et al., 2013) the investigation on the coordination of active front steering and rear braking for a driver-assist 
system in yaw control of a motor vehicle was proposed. In (Güvenç et al., 2009) asymmetrical load in a vehicle, combined with $\mu$-split braking forces caused by side wind or unilateral tire pressure loss was examined. As a result, unexpected yaw disturbances were observed, which required yaw stabilisation provided either by a driver or by an automatic driver-assist system.

Stability was also examined in relation to control systems. In (Segawa et al., 2001) the so-called $D^{*}$ control system was proposed, which, according to the authors, could offer mainly high steering response and reduced driving effort, whereas in (Zheng et al., 2006) the use of the vehicle yaw moment and wheel slip control were used in the dynamic control system, mainly for tracking the desired vehicle behaviour.

Taking the above into consideration, it is fair to say that research on the vehicle stability has two major directions: vehicle dynamics with the use of mechanical dependencies and control with the use of systems basing on the obtained data. It seems however important that the mechanical reliances could be connected with simulation results and normalized descriptions (ISO).

Analysis of the possibility to compare results obtained in a simulation with the ISO standards was presented in (Kisilowski and Zalewski, 2015). However, it was not previously discussed on specific examples.

In this paper, the motor vehicle model, considered as well in (Kisilowski and Zalewski, 2016), is used as an example. It seems questionable when, under what circumstances, a vehicle after accident and repair is roadworthy. In order to provide the realism of the analysed problem, the stochastic technical stability definition is taken into consideration, as it provides random disturbances acting on the mechanical system (here the vehicle). One of the discussed examples was previously created in (Kisilowski and Zalewski, 2016).

\section{Stability of a motor vehicle}

The concept of stability in terms of motor vehicle motion is presented in ISO 8855: 1991. To so called steady-state of the vehicle is described as a condition in which the sum of all external and inertial forces and moments forms a constant. Other conditions have been described as other states (non-steady). Hence, definitions of stability, quoted in the paper (Kisilowski and Zalewski, 2016), are as follows:

- non-periodic stability - stability characteristic at a prescribed steady-state equilibrium if, following any small and temporary disturbance or control input, the vehicle will return to the steady-state equilibrium without oscillation;

- neutral stability - stability characteristics at a prescribed steady state equilibrium if, for any small and temporary disturbance or control input, the vehicle motion remains close to the motion defined by the steady-state equilibrium, but does not return to it;

- oscillatory stability - oscillatory vehicle response of a decreasing amplitude and a return to the original steady-state equilibrium;

- non-periodic instability - ever-increasing response without oscillation;

- oscillatory instability - oscillatory response of an increasing amplitude about the initial steady-state equilibrium.

\section{Stochastic technical stability}

Stochastic technical stability is defined according to, e.g. (Bogusz, 1972), where a system of stochastic equations is assumed

$$
\frac{d x}{d t}=f(x, t, \xi(t))
$$


where $x=\left(x_{1}, \ldots, x_{n}\right)$ and $f(x, t, y)=\left(f_{1}, \ldots, f_{n}\right)$ and $\xi(t)=\left(\xi_{1}, \ldots, \xi_{n}\right)$ are vectors and $\xi(t)$ for $t \geqslant 0$ is a stochastic process which describes random disturbances. Any set of equations can be represented by such a system (Kisilowski and Zalewski, 2016).

The function $f(x, t, y)$ is assumed to be determined for each $x \in E_{n}, y \in E_{n}$ and $t \geqslant 0$. Absolute integrability is assumed for a stochastic process $f(0, t, \xi(t))$

$$
P\left\{\int_{0}^{T}|f(0, t, \xi(t))| d t<\infty\right\}=1 \quad \text { for each } \quad T>0
$$

as well as the existence of another stochastic process $f(X, t, \xi(t))$, which fulfills the Lipschitz criterion in $[0, T]$ interval for another process $\eta(t)$, also absolutely integrable in that given interval. This assumption is described as follows

$$
\left|f\left(x_{2}, t, \xi(t)\right)-f\left(x_{1}, t, \xi(t)\right)\right| \leqslant \eta(t)\left|x_{2}-x_{1}\right|
$$

As a result of the above assumptions, there exists only one solution of the set of equations (3.1), being an absolutely continuous stochastic process with the probability 1 for $t \geqslant t_{0}$, with the initial conditions $t=t_{0}$ and $x\left(t_{0}\right)=x_{0}$.

It is also assumed that there exist two areas in the Euclidean space $E_{n}: \omega$-limited and open and $\Omega$ - limited and closed, where $\omega \subset \Omega$. There is as well a positive number $\varepsilon$, where $0<\varepsilon<1$ and the stochastic process $X(t)$ specified for $t \geqslant t_{0}$.

Initial conditions for each solution are $t=t_{0}, x\left(t_{0}\right)=x_{0}$ and the solution itself is presented as $\left(t, t_{0}, x_{0}\right)$.

Definition of the stochastic technical stability reads: if every solution of equation (3.1), with its initial conditions belonging to $\omega$, remains in the $\Omega$ area with the probability $1-\varepsilon$, then system (3.1) is stochastically technically stable towards $\omega, \Omega$ and the process $\xi(t)$ with the probability $1-\varepsilon$ (Fig. 1).

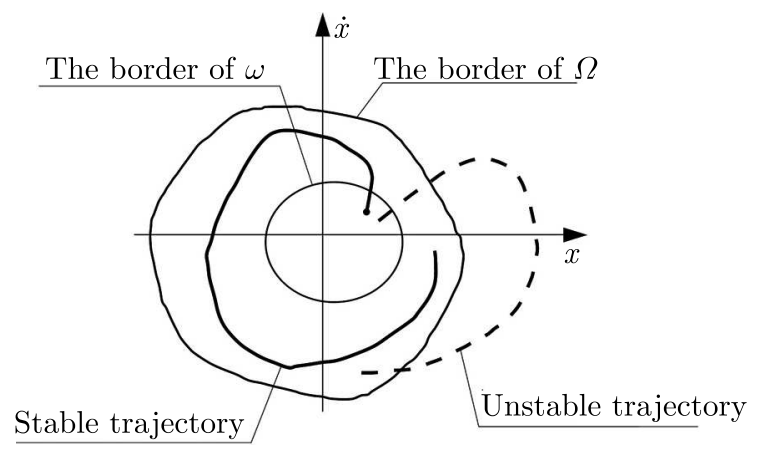

Fig. 1. Graphic interpretation of the stochastic technical stability. Source: Bogusz (1972)

It can also be described by the formula

$$
P\left\{\left(t, t_{0}, x_{0}\right) \in \Omega\right\}>1-\varepsilon \quad \text { for } \quad \bar{x}_{0} \in \omega
$$

The above definitions of the motor vehicle stability according to ISO standards can be compared with the stability defined for mathematical models, according to certain assumptions and criteria which concern not only the vehicle but the disturbances coming from road irregularities as well. It was previously theoretically discussed in (Kisilowski and Zalewski, 2015) but with no specific examples. As a result, the following conclusions were made in relation to the stochastic technical stability versus the ISO standards. All comparisons are possible if trajectories of the vehicle are taken into account. 
The definition of non-periodic stability can be compared with the stochastic technical stability provided that the accepted disturbances will generate a relatively small deviation from the assumed stable motion, because the return to this state should seem fluent without oscillations. In the presented case, random disturbances could, for example, originate from transverse irregularities of the road as well as a strong blow of wind whose resultant force would be perpendicular to the plane of symmetry of the vehicle. This may cause transverse drift of the vehicle.

The neutral stability (motion close to the steady-state, but without oscillations) can as well be compared to the stochastic technical stability, because of the assumption that the solution remains in the acceptable area around the steady-state.

The comparison seems also be possible between the oscillatory stability and the stochastic technical stability, since both definitions provide return of a representative point of the vehicle to the steady-state. Also implementing stochastic disturbances that cause oscillations seems important when considering the vehicle response. Oscillatory stability does not have to mean a periodic decrease of the trajectory amplitude.

Both periodic and non-periodic instabilities characterised by the strengthening of the vehicle response with or without oscillation, respectively, can be compared to the stochastic technical stability depending, among others, on which the model is analysed (linear or non-linear) and what type of disturbances are taken into account (constant or random).

\section{Comparison of the methods}

The above conclusions show that stability of technical objects presented as mathematical models with the use of differential equations (in this case motor vehicles) seems to be a valuable tool for analysis of the correctness of their functioning. Moreover, significant seems the fact of the possibility of experimental verification of tested models by analysing motion of their real counterparts.

In this Section, certain examples of comparison between the stochastic technical stability and selected ISO standards are shown. Three presented examples show simulation results for a sports two seater (in MSC Adams/Car environment), differently laden and moving in different road conditions. Description of this model was presented, among others, in (Zalewski, 2014a,b). In each given case the FTIRE (flexible tire ring) tire model was used instead of the PAC89 originally attached to the vehicle model, because of random irregularities in the road surface. Typical tire models do not allow running simulations on two-dimensional stochastic uneven road profiles, where the wavelength of a single irregularity is less than the wheel radius (Zalewski, 2014a,b).

Nominal parameters of the considered model of the motor vehicle in MSC Adams/Car have been adopted as in (Zalewski, 2014a,b):

- mass of the unladen vehicle: $m_{U B}=995 \mathrm{~kg}$;

- location of the "origo" point in relation to the front edge center of the unladen vehicle: $x_{c}=0.9 \mathrm{~m}, y_{c}=0 \mathrm{~m}, z_{c}=0.48 \mathrm{~m}$;

- nominal location of the center of mass in relation to the "origo" (Kisilowski and Zalewski, 2016) in the unladen vehicle: $x_{c}=1.5 \mathrm{~m}, y_{c}=0, z_{c}=0.45 \mathrm{~m}$;

- nominal values of the inertia moments for the unladen vehicle relative to the axes which intersect the "origo" point: $I_{x}=401 \mathrm{~kg} \cdot \mathrm{m}^{2}, I_{y}=2940 \mathrm{~kg} \cdot \mathrm{m}^{2}, I_{z}=2838 \mathrm{~kg} \cdot \mathrm{m}^{2}$;

- nominal values of the deviation moments of inertia of the unladen vehicle relative to the same intersecting "origo": $I_{x y}=0, I_{x z}=671 \mathrm{~kg} \cdot \mathrm{m}^{2}, I_{y z}=0$. 


\section{Example I}

As the first example, results from the work (Zalewski, 2014) are presented, where a single lane change maneuver has been simulated using the model described previously as well in the MSC Adams/Car component.

Assumptions for the vehicle load and the course of maneuver have been taken as follows:

- mass of the vehicle was increased by the baggage $\left(m_{B}=60 \mathrm{~kg}\right)$, driver $\left(m_{1}=75 \mathrm{~kg}\right)$ and passenger $\left(m_{2}=110 \mathrm{~kg}\right)$;

- initial speed of the vehicle moving at the fifth gear was $100 \mathrm{~km} / \mathrm{h}$;

- coordinates of the center of mass in the laden vehicle with respect to "origo": $x_{c L}=1.472 \mathrm{~m}, y_{c L}=0.085 \mathrm{~m}, z_{c L}=0.454 \mathrm{~m}$;

- inertia and deviation moments of inertia with regard to "origo": $I_{x L}=464 \mathrm{~kg} \cdot \mathrm{m}^{2}$, $I_{y L}=3442 \mathrm{~kg} \cdot \mathrm{m}^{2}, I_{z L}=3295 \mathrm{~kg} \cdot \mathrm{m}^{2}, I_{x y L}=155 \mathrm{~kg} \cdot \mathrm{m}^{2}, I_{x z L}=828 \mathrm{~kg} \cdot \mathrm{m}^{2}, I_{y z L}=$ $47.8 \mathrm{~kg} \cdot \mathrm{m}^{2}$.

The above mentioned "origo" point is the origin of the coordinate system located underneath the vehicle model (on the ground level) but moving along with the model during simulation. It is presented in Fig. 2.

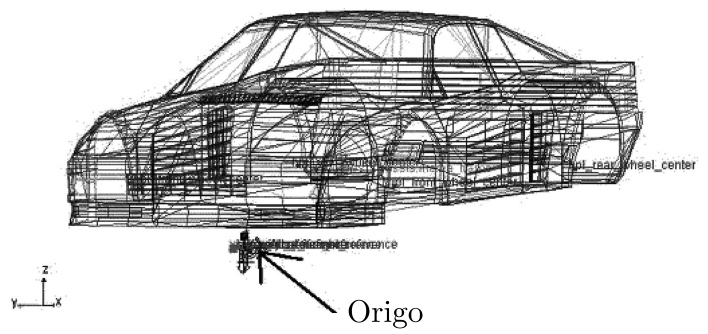

Fig. 2. Location of the "origo" point. Source: MSC Adams/Car

The vehicle model used here had non-linear elastic - damping elements. In Fig. 3, the spring deflection, whereas in Fig. 4 - the damping force versus velocity of the acting damper are presented. A MacPherson column based suspension is used in the model.

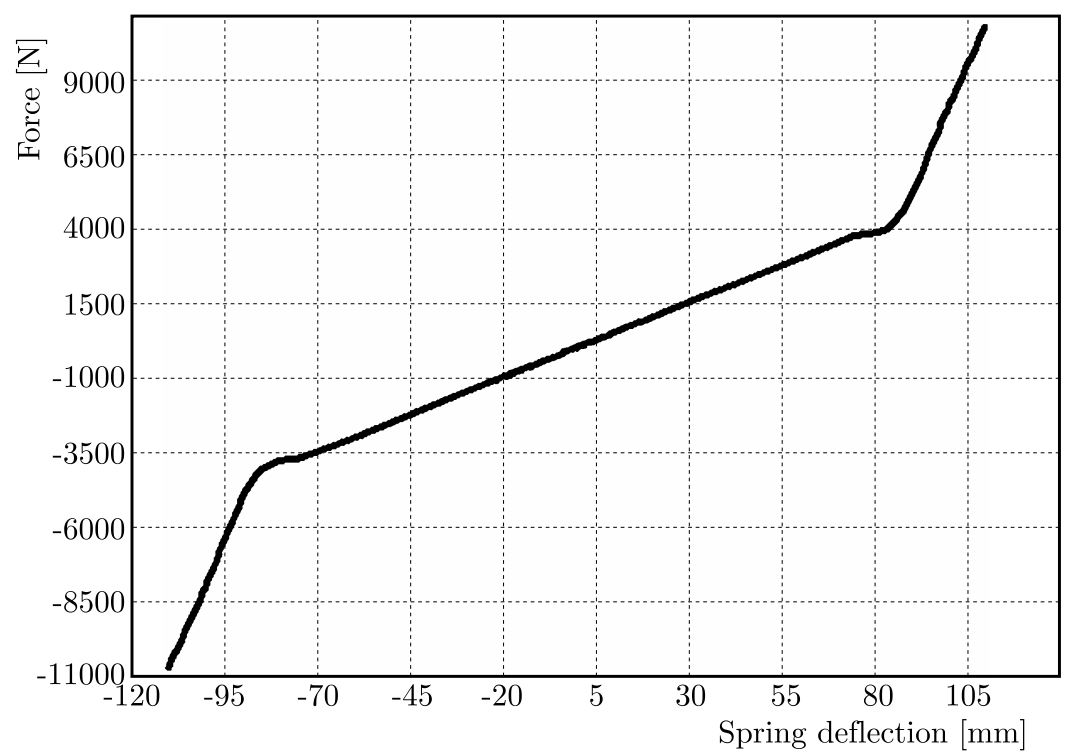

Fig. 3. The force - spring deflection characteristics in the vehicle suspension. Source: Zalewski (2014a) 


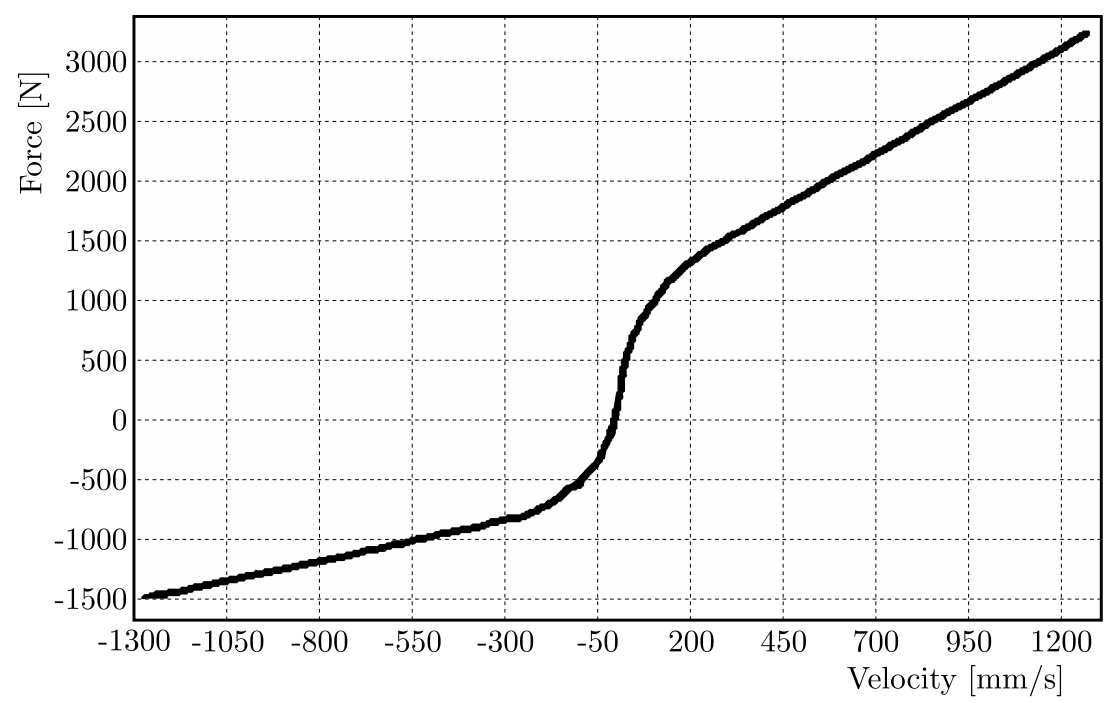

Fig. 4. The damping force - damper velocity characteristics in the vehicle suspension.

Source: Zalewski (2014a)

A single lane change maneuver simulation has been carried out for the following configurations:

a) vehicle laden as shown above, riding on a flat and icy road $(\mu=0.3)$;

b) vehicle laden as shown above and riding on an icy and randomly uneven road.

The maneuver has been realised on a $480 \mathrm{~m}$ long road section at a constant speed of $100 \mathrm{~km} / \mathrm{h}$. Random disturbances acting on the vehicle as road irregularities were described among others in (Zalewski, 2014a). The discussed maneuver has been carried out for extreme icy road conditions.

As a result, two trajectories were obtained, each for one of the above described configurations. The thin curve marks the vehicle trajectory on a flat surface, whereas the bold curve - the trajectory obtained for the uneven road (Fig. 5).

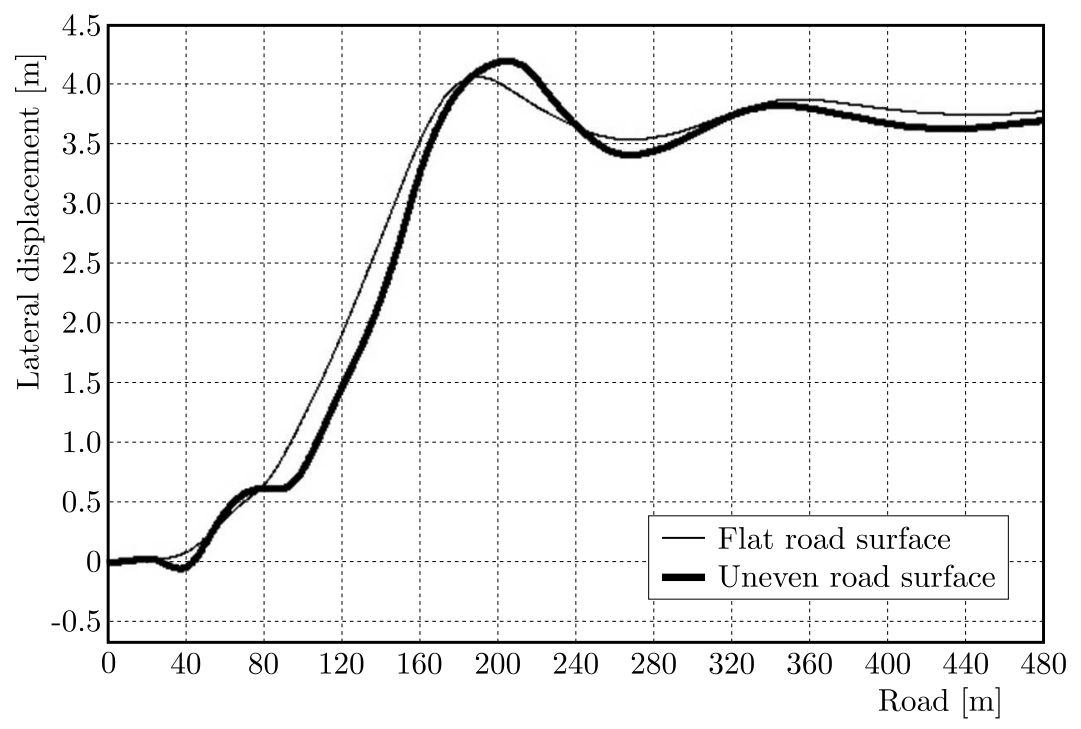

Fig. 5. Lateral displacement versus covered distance. Source: Zalewski (2014a) 


\section{Example II}

Another example is based on the results obtained from (Zalewski, 2014). By adding mass of the driver $\left(m_{1}=75 \mathrm{~kg}\right)$, passenger $\left(m_{2}=105 \mathrm{~kg}\right)$ and baggage $\left(m_{B}=50 \mathrm{~kg}\right)$, the entire mass of the vehicle model increased (Figs. 6 and 7), so that new parameters of mass - inertia in the vehicle body were determined:

- disturbed location of the mass center with regard to the "origo": $x_{c L}=1.481 \mathrm{~m}$, $y_{c L}=0.081 \mathrm{~m}, z_{c L}=0.454 \mathrm{~m}$;

- inertia moments of the vehicle with regard to the axes intercecting the "origo": $I_{x L}=461 \mathrm{~kg} \cdot \mathrm{m}^{2}, I_{y L}=3441 \mathrm{~kg} \cdot \mathrm{m}^{2}, I_{z L}=3297 \mathrm{~kg} \cdot \mathrm{m}^{2}$

- deviation moments of the vehicle with regard to the axes intersecting the "origo": $I_{x y L}=148 \mathrm{~kg} \cdot \mathrm{m}^{2}, I_{x z L}=824 \mathrm{~kg} \cdot \mathrm{m}^{2}, I_{y z L}=45.5 \mathrm{~kg} \cdot \mathrm{m}^{2}$.

Simulation of the impulse steer maneuver described in (Zalewski, 2014a) was carried, among others, for the icy even and icy, uneven road surface with randomly occurring irregularities. The vehicle moved at the speed of $70 \mathrm{~km} / \mathrm{h}$, and its course was disturbed by implementing an impulse turn of the steering wheel about $90^{\circ}$ counterclockwise.

The spring - damping elements were non-linear, similarly as in the first example, so the results depended on initial conditions and concerned only such cases as discussed in this paper.

Two vehicle configurations were selected for analysis: the laden vehicle on an icy flat road surface and the laden vehicle on an icy road surface with randomly appearing irregularities.

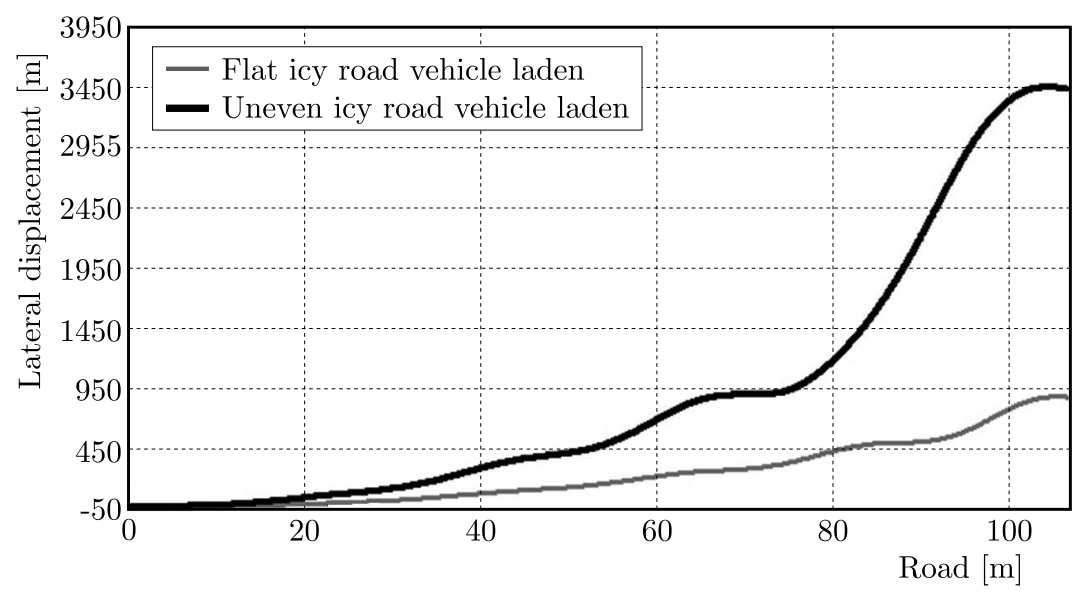

Fig. 6. The $\Omega$ area of the feasible solutions containing parts of the obtained trajectories.

Source: Zalewski (2014a)

The simulation took place on a $107 \mathrm{~m}$ length section of a straight road. Two trajectories for the described motion conditions were selected for further analysis - both representing the above discussed configurations, respectively (Fig. 6).

\section{Example III}

According to the work (Kisilowski and Zalewski, 2016), the following configuration of disturbances has been assumed:

- the vehicle was laden with the driver $\left(m_{d}=74 \mathrm{~kg}\right)$, passenger $\left(m_{p}=105 \mathrm{~kg}\right)$ and baggage $\left(m_{b}=45 \mathrm{~kg}\right)$.

As in the previous examples, new coordinates of the center of mass have been determined:

- location of the center of mass for the laden vehicle in regard to the "origo": $x_{c L}=1.562 \mathrm{~m}$, $y_{c L}=0.016 \mathrm{~m}, z_{c L}=0.471 \mathrm{~m}$; 
- inertia moments of the laden body with regard to the axes intersecting the "origo": $I_{x L}=460 \mathrm{~kg} \cdot \mathrm{m}^{2}, I_{y L}=3624 \mathrm{~kg} \cdot \mathrm{m}^{2}, I_{z L}=3464 \mathrm{~kg} \cdot \mathrm{m}^{2}$;

- deviation moments of the laden body with regard to the axes intersecting the "origo": $I_{x y L}=29 \mathrm{~kg} \cdot \mathrm{m}^{2}, I_{x z L}=863 \mathrm{~kg} \cdot \mathrm{m}^{2}, I_{y z L}=8.8 \mathrm{~kg} \cdot \mathrm{m}^{2}$.

An incomplete double lane change maneuver has been simulated. After the maneuver was finished, the vehicle remained in the middle of width of a single-lane road. The vehicle drove at the speed of $120 \mathrm{~km} / \mathrm{h}$, reflecting the necessity of an emergency response to an emerging obstacle. The simulation was carried out in MSC Adams/Car component as well in two configurations:

- laden vehicle moving on a dry and flat road;

- laden vehicle on an icy and randomly uneven road.

The resulting two trajectories for the described case are shown in Fig. 7.

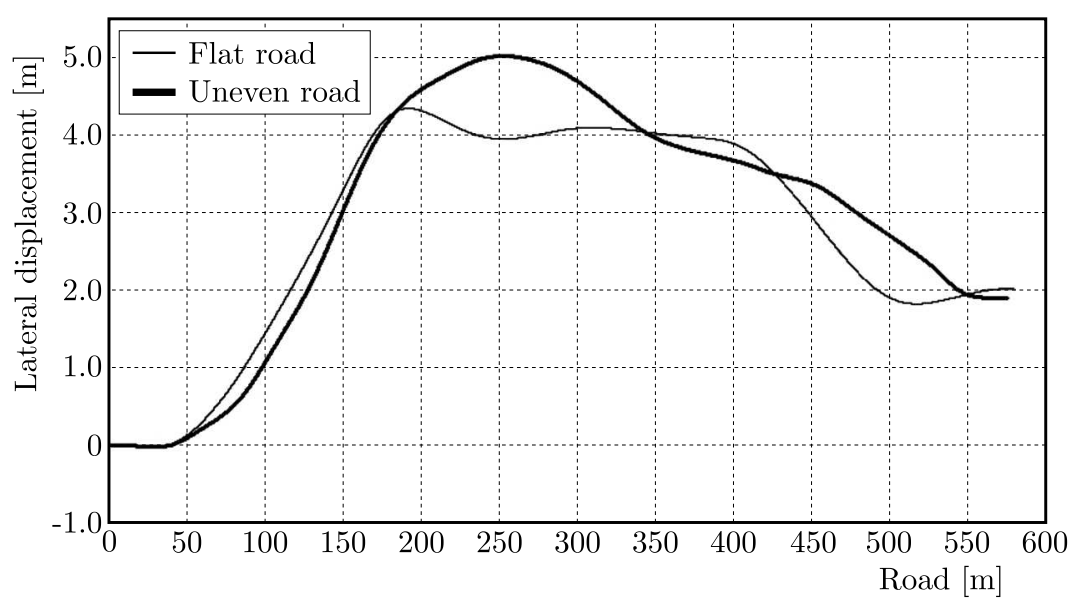

Fig. 7. Lateral displacement of the vehicle model versus the distance covered, for the laden vehicle body and both road surface conditions. Source: Kisilowski and Zalewski (2016)

Basing on the obtained simulation results for three different maneuvers in different road conditions, certain conclusions can be made. Provided that such analysis can be conducted on the basis of the obtained trajectories, it seems possible to compare the stochastic technical stability with the stability according to ISO standards, both for the motor vehicle in virtual and real world, respectively.

As in Example I, it can be seen that both trajectories in Fig. 5, after performing the single lane change, remain close to each other. If the thin line was assumed to be the steady state motion, than the thick line could be regarded as neutrally stable, according to the ISO definitions. It seems that the qualitative assesment of both obtained trajectories is sufficient in the given case.

In Example II, however, it seems that the vehicle realising motion along the thick line is nonperiodically unstable versus the thin line, assumed to be the steady state motion. For the first $100 \mathrm{~m}$ of the covered distance, the difference between the lateral displacement of both trajectories for the horizontal axis (distance [m], Fig. 6) increased without oscillation, as according to the ISO standards.

Finally, for the trajectories obtained in Example III (Fig. 7), it seems not easy to justify whether the thick line is oscillatory or neutrally stable versus the thin line. However, none of the given examples proved to be oscillatory unstable or non-periodic stable (none of them returned to the assumed steady state). 


\section{Conclusions}

Basing on the presented results, certain conclusions can be made. It seems that comparison between stability of vehicles examined in virtual reality with the use of the given mathematical definition, and stability as a feature of real vehicles as mechanical objects described according to the specified norm, is possible. Moreover, the presented method seems versatile enough to be used in other examples, i.e. different vehicle models and different maneuvers.

Correctness of the final results in this case seems to depend on the accuracy of the vehicle model as well as simulation of its motion. However, vehicle stability, considered as a certain feature of each vehicle in the period of its maintenance, does not seem to require vehicle models being as accurate as the real vehicles. The ability to specify the mass - inertia parameters of the modeled vehicle seems to be the most important.

\section{References}

1. Bogusz W., 1972, Technical Stability (in Polish), PWN, Warszawa

2. Chung T., Yi K., 2006, Design and evaluation of side slip angle-based vehicle stability control scheme on a virtual test track, IEEE Transactions on Control Systems Technology, 14, 2

3. Doumiati M., Sename O., Dugard L., Martinez-Molina J.J., Gaspar P., Szabo Z., 2013, Integrated vehicle dynamics control via coordination of active front steering and rear braking, European Journal of Control, 19

4. Güvenç B.A., Güvenç L., Karaman S., 2009, Robust yaw stability controller design and hardware-in-the-loop testing for a road vehicle, IEEE Transactions on Vehicular Technology, 58, 2

5. Karnopp D., 2004, Vehicle Stability, CRC Press

6. Khasminskit R., 2012, Stability of Stochastic Differential Equations, 2nd Edition, Springer-Verlag, Berlin, Heidelberg

7. Kisilowski J., Zalewski J., 2008, Chosen problems of examination of car stability, Archives of Transportation Systems Telematics, 1, 1

8. Kisilowski J., Zalewski J., 2015, Stability of road vehicle mathematical models and the real objects (the ISO norm) (in Polish), Logistyka, 4

9. Kisilowski J., Zalewski J., 2016, Analysis of the stochastic technical stability of engineering structures on example of moving car, Journal of Theoretical and Applied Mechanics, 54, 4

10. Segawa M., Nakano S., Nishirara O., Kumamoto H., 2001, Vehicle stability control strategy for steer by wire system, JSAE Review, 22

11. ZALeWski J., 2014a, The influence of road conditions on the stability of a laden vehicle mathematical model, realising a single lane change maneuver, Telematics - Support for Transport, Communications in Computer and Information Science, 471

12. ZALEWSKI J., 2014b, Effect of disturbing the center of gravity on stability of a vehicle subject to impulse distortion of the straightforward motion (in Polish), Zeszyty Naukowe Polskiego Stowarzyszenia Zarzadzania Wiedza, 70

13. Zheng S., Tang H., Han Z., Zhang Y., 2006, Controller design for vehicle stability enhancement, Control Engineering Practice, 14 